\title{
La escuela como orientadora y promotora de inclusión La experiencia de formar para la vida y el trabajo en la educación secundaria (Córdoba, Argentina)
}

\author{
THE SCHOOL LEADING TO AND PROMOTING INCLUSION.THE EXPERIENCE OF TRAINING HIGH SCHOOL \\ STUDENTS FOR LIFE AND WORK (CÓRDOBA, ARGENTINA) \\ A ESCOLA COMO ORIENTADORA E PROMOTORA DE INCLUSIÓN. \\ A EXPERIÊNCIA DE FORMAR PARA A VIDA E O TRABALHO NA EDUCAÇÃO SECUNDÁRIA (CÓRDOBA, ARGENTINA)
}

\section{Horacio Ademar Ferreyra* / hferreyra@coopmorteros.com.ar}

\section{Resumen}

Los complejos escenarios actuales desafían los procesos de construcción, desarrollo e implementación curricular a que propongan nuevas maneras de hacer escuela para los jóvenes de hoy. Esto implica transformaciones en los saberes prioritarios y en las perspectivas, interpretaciones y prácticas educativas. En este marco, la propuesta del espacio curricular "Formación para la Vida y el Trabajo", contemplado en la oferta educativa de la Educación Secundaria Obligatoria del Ministerio de Educación de la Provincia de Córdoba, Argentina, delinea un currículum que, articulando educación, mundo de la vida y mundo del trabajo, promueve nuevos modos de relación con el saber socialmente relevante y con la diversificación de los ámbitos de experiencia escolar y extraescolar de los estudiantes.

\section{Summary}

The complex present scenarios urge the processes of curricular construction, development and implementation to suggest new ways of educating our present teenagers. This implies a transformation not only in the top priority knowledge but also in the perspectives, interpretations and educational practices. Within this framework, the proposal of the subject Training for Life and Work, considered in the educational offer of Compulsory High School Education of the Ministry of Education in the Province of Córdoba in Argentina, formulates a curriculum which by means of the articulation between education, the life realm and the work realm will foster new relationships with the socially relevant knowledge and the diversification of the in-school and out-of-school experiences in our students.

\section{Resumo}

Os complexos palcos actuais desafiam os processos de construção, desenvolvimento e implementación curricular a que proponham novas maneiras de fazer escola para os jovens de hoje. Isto implica transformações nos saberes prioritarios e nas perspectivas, interpretações e práticas educativas. Neste marco, a proposta do espaço curricular "Formação para a Vida e o Trabalho", contemplado na oferta educativa da Educação Secundária Obrigatória do Ministério de Educação da Província de Córdoba, Argentina, delinea um currículum que, articulando educação, mundo da vida e mundo do trabalho, promove novos modos de relação com o saber socialmente relevante e com a diversificación dos âmbitos de experiência escolar e extraescolar dos estudantes.
Palabras clave

Educación secundaria, currículo, vida, trabajo, prácticas educativas.

Keywords

High school, curriculum, life, work, educational practices.

Palavras chave

Educação secundária, currículo, vida, trabalho, práticas educativas.

* Licenciado en Ciencias de la Educación y Doctor en Educación (Universidad Católica Córdoba UCC). Diplomado y Especialista en Currículo y prácticas en contextos; Diplomado en Gestión y Conducción del Sistema Educativo y sus Instituciones (FLACSO Argentina). Posdoctorado en Ciencias Sociales (Universidad Nacional de Córdoba UNC-CEA, Argentina y Universidad Autónoma Metropolitana, unidad Xochimilco -UAMxMéxico). Subsecretario de Estado de Promoción de Igualdad y Calidad Educativa del Ministerio de Educación de la Provincia de Córdoba (ME). Docente e Investigador de la UCC. Docente e Investigador invitado en la USTA (Colombia). Se desempeñó como Director General de Educación Media y Superior (ME Córdoba, Argentina). Capacitador y consultor educativo en el nivel provincial, nacional e internacional. 


\section{Introducción ${ }^{1}$}

Ofrecer a los jóvenes una educación de calidad, supone no sólo generar dispositivos y estrategias que faciliten el acceso, permanencia y terminalidad de la escuela, o proveer de recursos a las instituciones. Se trata de construir propuestas curriculares que conviertan el tránsito por la Educación Secundaria en: "una experiencia de aprendizaje integral, sustantiva, que sirva para la vida y para el trabajo" (Jacinto, 2009). Es ésta la verdadera inclusión educativa a la que todos los jóvenes tienen derecho y a la que el Estado está obligado.

Desde estas convicciones y en el marco de las finalidades formativas de la Educación Secundaria en Argentina², la transformación curricular de la Educación Secundaria en la provincia de Córdoba ${ }^{3}$, República Argentina, definió un nuevo espacio curricular ${ }^{4}$-Formación para la Vida y el Trabajo- con la intención de contribuir a que los estudiantes puedan pensar sus procesos de participación social, política, cultural y económica en el marco de una

1 Se recuperan aquí ideas desarrolladas por el autor en la Conferencia pronunciada en el V Congreso Internacional "Cultura del Trabajo: Jóvenes y Valores", organizado en 2009 por Inclusión Social Sustentable, y en el artículo "Vida y Trabajo como espacio de Formación en la Educación Secundaria en la Provincia de Córdoba, Argentina" (en proceso de evaluación, Revista Iberoamericana sobre Calidad, Eficacia y Cambio en Educación -REICE-).

2 Según lo establecido por la Ley de Educación Nacional Nº 26.206/2006, Art. 30 que, se refiera a -"habilitar a los/las adolescentes y jóvenes para el ejercicio pleno de la ciudadanía, para el trabajo y para la continuación de los estudios"- y, específicamente, en la Ley de Educación de la Provincia de Córdoba No 9870, Art. 39 - "implementar, en el marco de los proyectos curriculares institucionales, procesos de orientación educacional que contribuyan a las elecciones vocacionales, vinculando a los alumnos con el mundo del trabajo, la producción, la ciencia y la tecnología"-.

3 La Educación Secundaria en la Provincia, atendiendo a lo establecido por las Leyes Nacional y Provincial de Educación vigentes, constituye una unidad pedagógica y organizativa destinada a los adolescentes y jóvenes de 11/12 a 17/18 años, que hayan cumplido con el nivel de Educación Primaria. La propuesta formativa se estructura en dos Ciclos: un Ciclo Básico - común a todas las orientaciones y modalidades - de tres años de duración, y un Ciclo Orientado -con carácter diversificado- de tres años para las ofertas de Educación Secundaria Orientada, y de cuatro, para las de Educación Secundaria Técnico Profesional y Artística. Las Orientaciones de la Educación Secundaria que se ofrecen en la Provincia son 10 (diez): Economía y Administración, Ciencias Sociales y Humanidades, Arte, Ciencias Naturales, Educación Física, Turismo, Informática, Comunicación, Lenguas, Agro y Ambiente.

4 Un espacio curricular delimita un conjunto de aprendizajes y contenidos educativos, provenientes de uno o más campos del saber, seleccionados para ser enseñados y aprendidos durante un período escolar determinado, fundamentado en criterios epistemológicos, pedagógicos, psicológicos, entre otros, y constituye una unidad autónoma de evaluación y acreditación. perspectiva de autonomía individual y colectiva ${ }^{5}$. De esta manera, se otorga a la escuela el rol de orientadora y promotora de procesos de inclusión. Es el objetivo de este trabajo presentar, describir y evaluar el espacio curricular mencionado.

\section{Intencionalidades formativas}

Garantizar el derecho a una educación de calidad para todos los jóvenes desde la perspectiva curricular, requiere de transformaciones en los enfoques, las interpretaciones y las prácticas (UNESCO, 2007). La construcción y el desarrollo curricular para la generación de condiciones educativas inclusivas y de calidad, se orientan a promover nuevos modos de relación con el saber socialmente relevante, buscan diversificar los ámbitos de experiencia de los estudiantes; abrir para ellos nuevos espacios de participación en el contexto y habilitar oportunidades para que, desde su presente, puedan comenzar a proyectarse en la dimensión vocacional y socio ocupacional a los fines de su efectiva inclusión social.

En este sentido, Formación para la Vida y el Trabajo se presenta como un espacio curricular orientado, primordialmente, a generar y fortalecer vínculos entre los saberes escolares y extraescolares; a producir articulaciones sustantivas entre la escuela y la comunidad y a promover la participación activa y transformadora de los jóvenes en los diversos escenarios sociales, acompañándolos en la ampliación de su perspectiva de conocimientos y relaciones y en la progresiva concreción de sus intereses y expectativas en proyectos personales y socialmente relevantes. Para ello, el espacio define una serie de aprendizajes y contenidos y prevé experiencias educativas tendientes a poner en marcha estrategias de formación para la vida y el trabajo, vinculadas con el ejercicio protagónico de la ciudadanía y con el desarrollo personal y social de los estudiantes.

\footnotetext{
5 Se inició su implementación en el año 2010-como experiencia piloto- en $3^{\circ}$ año, y a partir de allí, en los años posteriores continúa su desarrollo: 2011, en $4^{\circ}$ año; 2012, en $5^{\circ}$ y 2013 , en $6^{\circ}$ año. Cabe aclarar que se están desarrollando experiencias anticipadas de innovación en los contenidos y prácticas educativas de 6to, aún en el marco de la anterior estructura curricular de la Educación Media (en su último año de vigencia). Todo el proceso ha sido precedido de numerosas instancias de consulta y de validación en la práctica escolar, así como de variados dispositivos de capacitación docente y acompañamiento institucional que aún continúan.
} 
En línea con tales propósitos, el diseño curricular ha priorizado la apropiación de conocimientos y el desarrollo de habilidades, aptitudes y actitudes para la vida y el trabajo, a fin de que los estudiantes puedan desempeñarse con mayor potencial en su quehacer cotidiano, seguir aprendiendo, aplicar lo aprendido en forma continua y con crecientes niveles de autonomía a lo largo de la vida -saber hacer con saber y con conciencia sobre el impacto de ese hacer-, y enfrentando, de manera activa y responsable, como miembros de su comunidad, los retos que impone una sociedad en permanente cambio.

El diseño curricular de Formación para la Vida y el Trabajo define, entonces, una propuesta formativa que se caracteriza por:

- Articular aprendizajes relacionados con la participación ciudadana, la organización social, la intervención creativa en la comunidad, la consolidación de la condición de estudiante (con las capacidades, disposiciones y actitudes que implica), la indagación de la cultura del trabajo y sus dinámicas, la formulación y gestión de proyectos personales, grupales y comunitarios.

- Organizarse en torno a experiencias educativas que fomenten la conexión curricular puesto que -en ellas- los jóvenes tienen la posibilidad de integrar y articular los aprendizajes alcanzados en los distintos espacios curriculares y trayectos de formación. Esto les permite asumir el desafío de generar proyectos de acción que colaboren en la modificación de aspectos de la realidad escolar, local, regional.
- Destacar el valor formativo del trabajo cooperativo y de la participación en experiencias comunitarias de resolución de necesidades comunes, como ejercicio activo de la ciudadanía.

- Presentarse como una oportunidad para que los jóvenes se autoafirmen y desarrollen como sujetos a partir del vínculo con los otros, en el espacio grupal (Borzese, 2008).

- Crear ambientes favorables a la innovación y a la generación de aprendizajes colectivos, no sólo en el ámbito del aula y de la escuela, sino en los más diversos lugares de la vida.

- Incorporar de manera activa a las familias y comunidades de pertenencia.

- Desarrollar procesos de construcción conjunta interdisciplinarios e intersectoriales.

\section{Propuesta formativa}

El espacio curricular traza un trayecto formativo que se inicia en el último año del Ciclo Básico de la Educación Secundaria (3er año) y recorre, con diversos énfasis, todo el Ciclo orientado (4to, 5to y 6to año). En el siguiente cuadro, se sintetizan las particularidades e interrelaciones de los distintos componentes del trayecto formativo (énfasis, núcleos estructurantes, propuesta pedagógica y aprendizajes, y contenidos6).

6 En los aprendizajes previstos, quedan involucrados diversos tipos de contenidos: conceptos, formas culturales, lenguajes, valores, destrezas, actitudes, procedimientos y prácticas. 


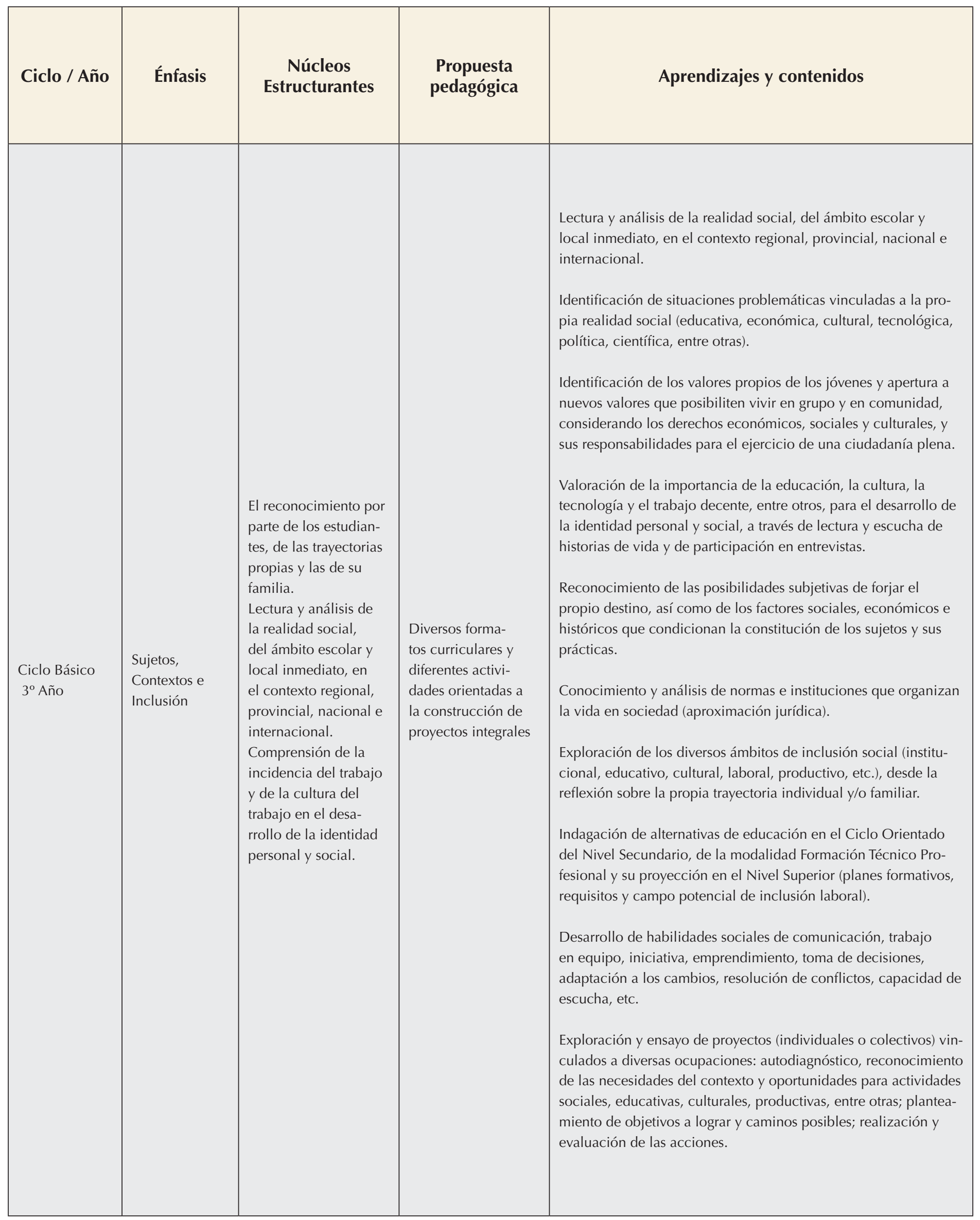




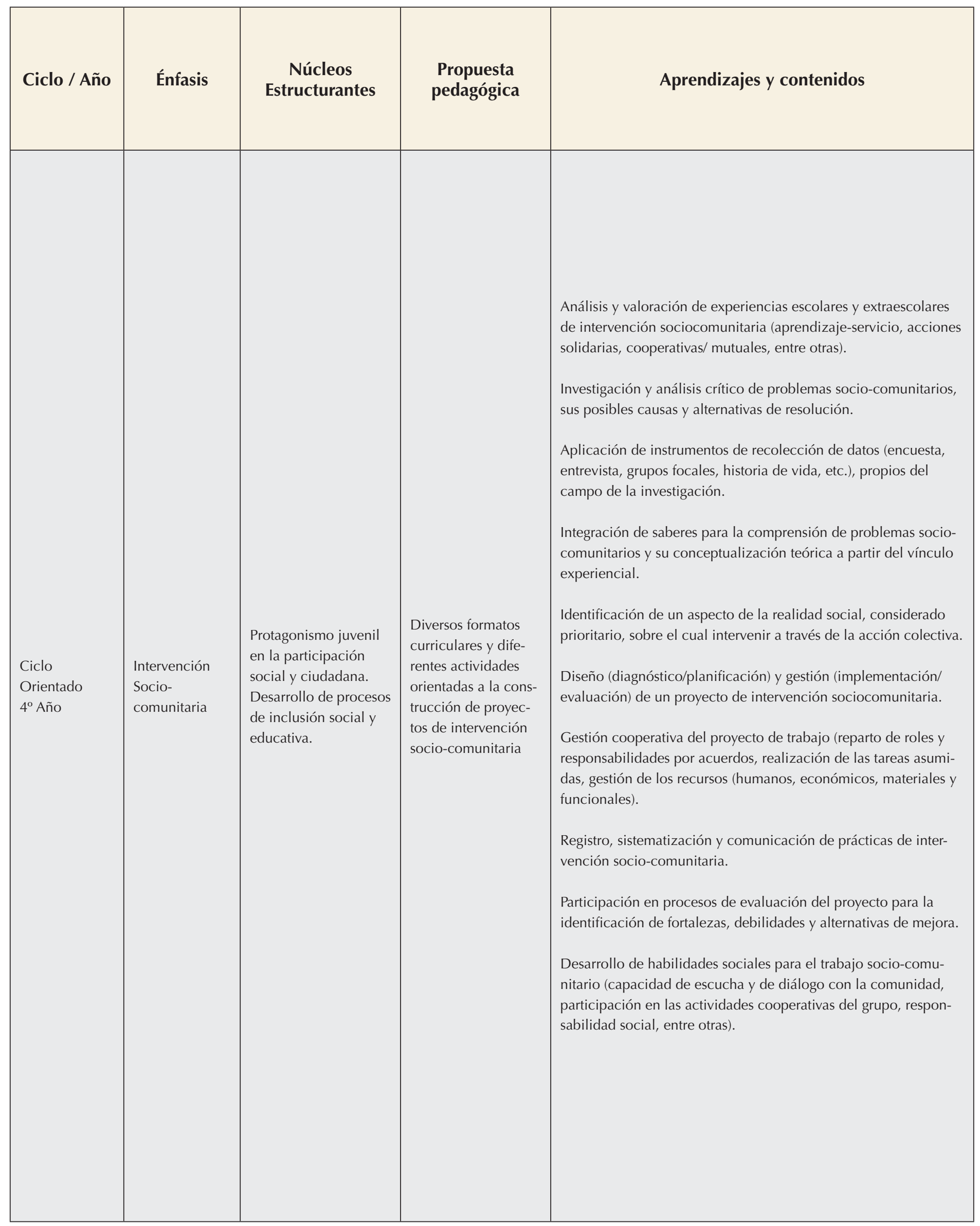




\begin{tabular}{|c|c|c|c|c|}
\hline Ciclo / Año & Énfasis & $\begin{array}{c}\text { Núcleos } \\
\text { Estructurantes }\end{array}$ & $\begin{array}{l}\text { Propuesta } \\
\text { pedagógica }\end{array}$ & Aprendizajes y contenidos \\
\hline $\begin{array}{l}\text { Ciclo } \\
\text { orientado } \\
5^{\circ} \text { Año }\end{array}$ & $\begin{array}{l}\text { Horizontes } \\
\text { Vocacionales y } \\
\text { Socio- ocupa- } \\
\text { cionales }\end{array}$ & $\begin{array}{l}\text { - Progresiva configu- } \\
\text { ración de proyectos } \\
\text { personales, vocaciona- } \\
\text { les, formativos y socio- } \\
\text { ocupacionales. } \\
\text { - Espacios y oportu- } \\
\text { nidades para cons- } \\
\text { truir conocimiento } \\
\text { situacional acerca del } \\
\text { ámbito de los estudios } \\
\text { superiores y del mun- } \\
\text { do del trabajo. }\end{array}$ & $\begin{array}{l}\text { Diversos formatos } \\
\text { curriculares y dife- } \\
\text { rentes actividades } \\
\text { orientadas a la cons- } \\
\text { trucción de proyec- } \\
\text { tos vocacionales y } \\
\text { socio- ocupacionales }\end{array}$ & 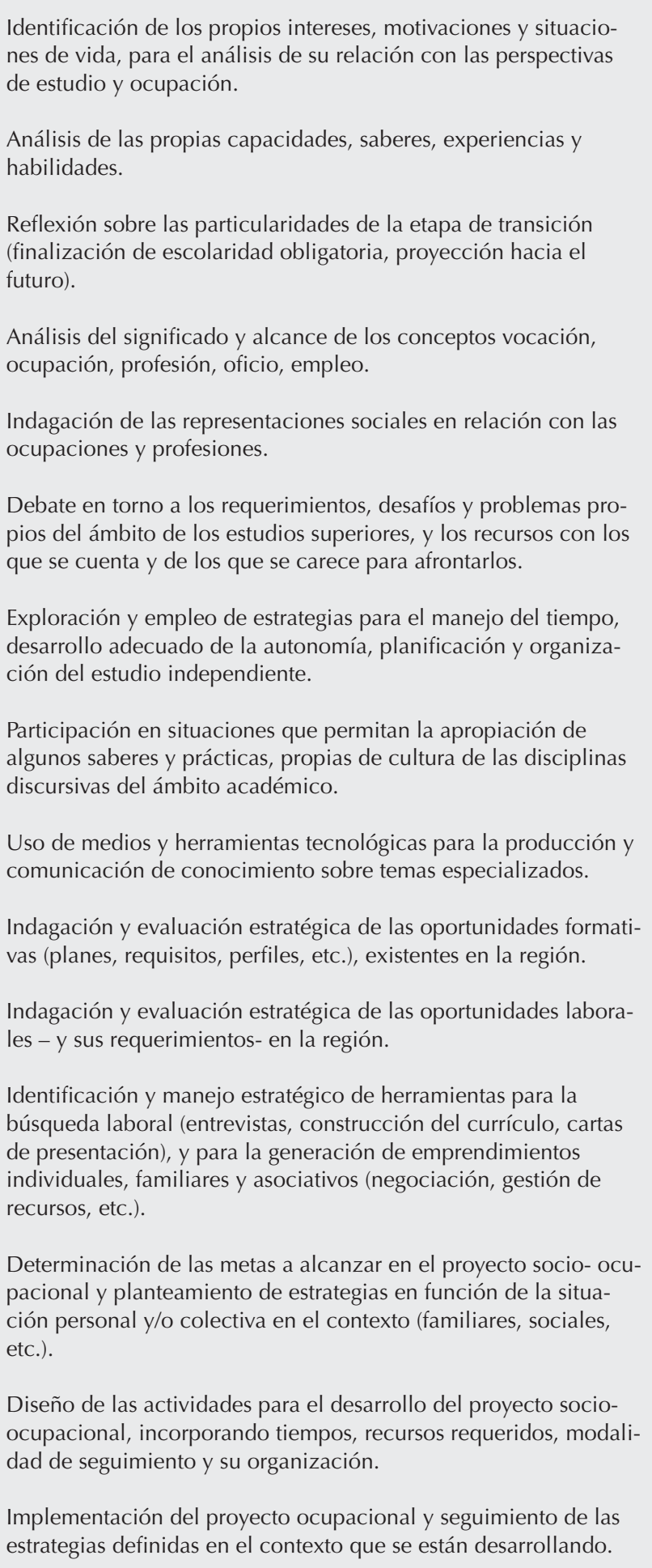 \\
\hline
\end{tabular}




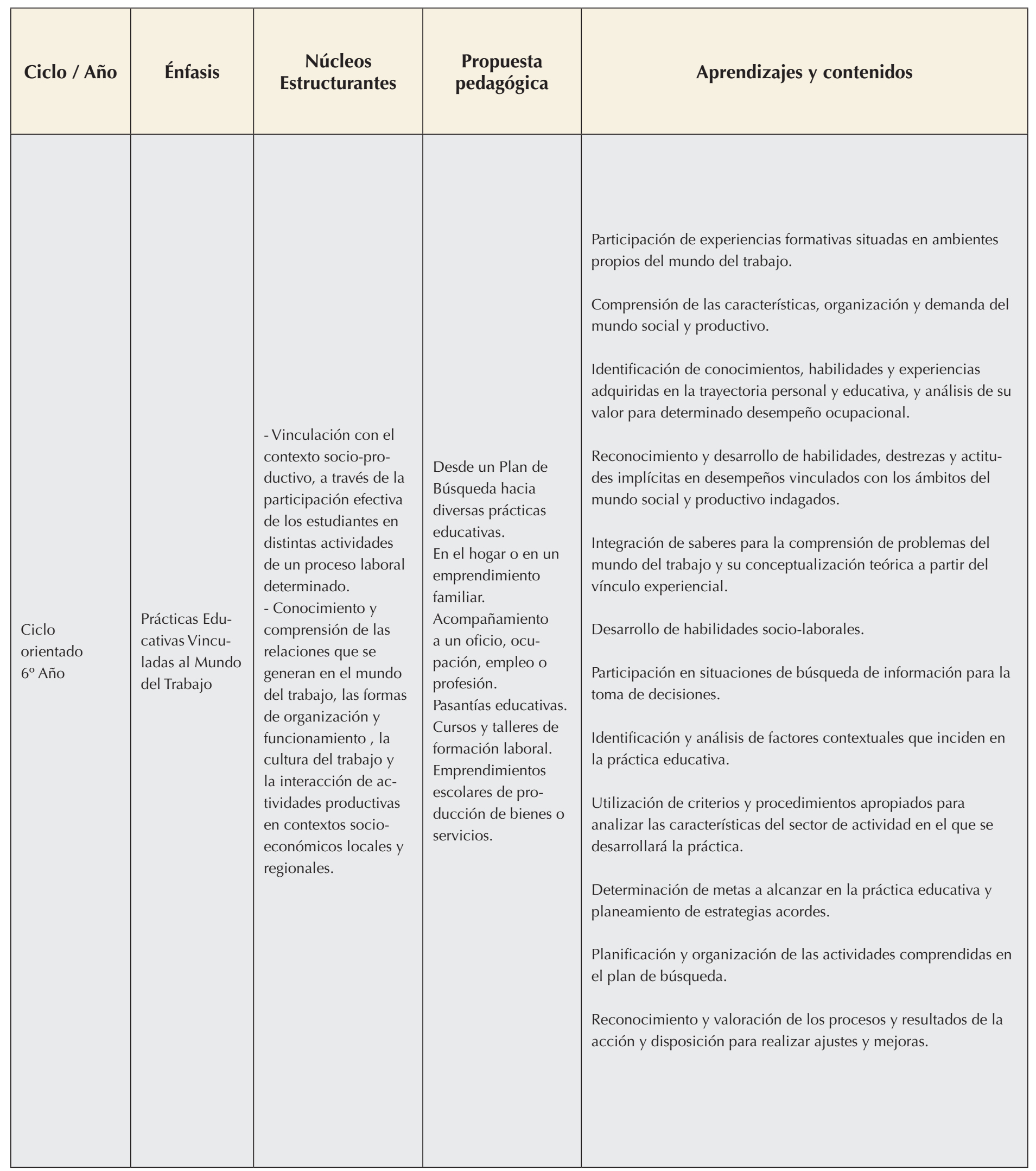

Fuente: Elaboración propia a partir de los Diseños Curriculares de Educación Secundaria Ciclo Básico (2011-2015) y Ciclo Orientado (2012-2015)- Tomos I a XVIII. (Gobierno de Córdoba, Argentina. Ministerio de Educación). 
Formación para la vida y el trabajo procura generar un espacio donde estén presentes las distintas realidades y concepciones de la adolescencia y la juventud, como construcciones sociales en la actualidad. Este proceso representa, para los docentes, un desafío significativo, que, entre otros aspectos, los enfrenta a la necesidad de partir de la comprensión del "ser adolescente y joven" en la actualidad, y los lleva, en primer término, a revisar las propias representaciones y a desarrollar, además, la capacidad de escucha y la aceptación de lo diverso, con el objeto de facilitar los vínculos necesarios para poder enseñar y aprender.

Las experiencias de implementación de este nuevo espacio curricular en la escuela y en las aulas, permiten visualizar propuestas de enseñanza que contemplan:

\section{A. Situaciones de aprendizaje organizadas en torno a la diversidad de formatos curriculares y pedagógicos $^{7}$}

Ellas posibilitan diversos modos de vinculación con el conocimiento, diferentes interacciones de los sujetos, flexibilización de tiempos, espacios y agrupamientos. Por ejemplo, y más allá del tradicional formato materia/ asignatura, se están desarrollando:

- Proyectos: que llevan a los estudiantes a involucrarse en tareas diversas, asumir distintos roles y desempeñar funciones diferentes, en favor de una meta común. En el marco de Proyectos Ocupacionales (individuales o colectivos), los adolescentes, luego de un análisis de sus características y de la situación de su contexto, toman decisiones y realizan una experiencia de diseño y gestión de un trayecto personal o colectivo, vinculado a lo educativo, lo laboral, productivo o sociocomunitario, entre otras posibilidades ${ }^{8}$.

Así, se están poniendo en marcha 1) Investigaciones escolares, a partir de problemáticas de interés (oferta de estudios secundarios en la región /

7 Para ampliar información relacionada con las características e implementación de los formatos curriculares, véase Anexo Opciones de Formatos Curriculares y Pedagógicos en Gobierno de Córdoba, 2011, pp. 28-41.

8 Este Proyecto, en sus distintas etapas o fases, debe permitir el abordaje integrado de la totalidad de aprendizajes y contenidos prescriptos, pero siempre desde una perspectiva situada en cuanto a la dimensión personal y social de los participantes y en un marco de respeto por sus decisiones. orientaciones que ofrecen las instituciones educativas, desarrollo económico local, condiciones de empleabilidad, entre otras); 2) Emprendimientos, fundamentalmente de carácter socio-comunitario, cooperativos, mutuales, orientados no solamente a actividades productivas, sino también a otras de carácter social, científico, artístico, cultural, deportivo, etc.; 3) Desarrollos tecnológicos, que demandan la elaboración y producción de algún objeto o su montaje (una máquina, un audiovisual, un terrario, un huerto escolar, un periódico, etc.); 4) Acciones de trabajo solidario, que posibilitan a los estudiantes, organizados y acompañados por docentes, una aproximación crítica a los problemas sociales y una implicación activa frente a ellos, en el marco de su formación como sujetos políticos capaces de comprometerse en la construcción de una sociedad más justa.

Para la puesta en marcha de los proyectos sociocomunitarios, se combinan tiempos en aula (desarrollo de aprendizajes y contenidos, reflexión, planeamiento) con tiempos específicos, destinados al desarrollo de la actividad comunitaria, cooperativa y solidaria en el mismo establecimiento, en locales de organizaciones comunitarias o de la sociedad civil, con las que se han establecido convenios institucionales, o en espacios comunitarios.

En todas estas experiencias, el protagonismo de los estudiantes se inicia desde la selección del proyecto y el planeamiento de la intervención hasta las instancias de evaluación procesual y sumativa de todo el proceso. Por otra parte, y con el propósito de que la intervención socio-comunitaria constituya una propuesta sólida y coherente, y de que los estudiantes cuenten con las herramientas necesarias, se ofrecen cursos o talleres de formación; por ejemplo, curso de promotores ambientales, talleres de narración oral y lectura en voz alta, curso de técnicas para documentación narrativa, entre otros. También se realizan consultas y entrevistas a referentes comunitarios, del ámbito de la cultura, científicos, otros profesionales, integrantes de ONGs, miembros de programas sociales, quienes aportan sus saberes y experiencias.

- Talleres: que favorecen un abordaje de contenidos y aprendizajes de un modo más directamente ligado al hacer y a la interactividad, con base en la experiencia personal y la de los otros, así como

12 nodos y nudos / volumen 4 N. 33 / julio-diciembre / 2012 ISSN: 0122-4328 / p.p 05-16 
en la experimentación y la producción. Las posibilidades que ofrece este formato están siendo capitalizadas por instituciones y docentes para organizar, por ejemplo: talleres de oralidad, lectura y escritura, de manejo y organización del tiempo (uso de agendas, calendarios, etc.), de formación laboral, de lectura y análisis de leyes, tratados, etc.

- Observatorios: para abordar el reconocimiento de las necesidades del contexto y de oportunidades para actividades sociales, educativas, culturales, productivas, entre otras. De esta manera, se favorece en los estudiantes el desarrollo de capacidades de búsqueda, recopilación, sistematización e integración de información necesaria y útil para la exploración y ensayo de proyectos (individuales o colectivos) vinculados a diversas ocupaciones. Así, por ejemplo, se indagan "las representaciones sociales en relación con las ocupaciones y profesiones", a través de encuestas y entrevistas, como así también mediante la publicación de contenidos en la prensa gráfica, radial, televisiva y digital.

- Seminarios: destinados a la profundización de ciertos contenidos curriculares, a través de la indagación en torno a ciertos temas o problemáticas, tales como los cambios en los procesos de trabajo: aspectos socio-históricos, científicos, tecnológicos, ético-políticos; las relaciones sociales en torno al trabajo, desigualdades y diversidades; los mercados del trabajo/mundo del trabajo, sus regulaciones, la protección laboral.

- Ateneos: que permiten el análisis y la discusión colectiva de casos relacionados con temas y problemáticas propios de los proyectos personales y sociales, el ingreso y permanencia en los estudios superiores, el mundo del trabajo.

\section{B. Diversidad de abordajes didácticos y actividades de aprendizaje}

La implementación de este espacio curricular, ha determinado la necesidad de ofrecer a los estudiantes experiencias de aprendizaje diversas; en tal sentido, un análisis de la planificación de los profesores y la observación de situaciones de aula, ha permitido identificar una ampliación y enriquecimiento de las actividades: entrevistas a referentes comunitarios, estudio de situaciones, trabajo exploratorio y problema- tizador, jornadas de profundización temática en torno a tópicos formativos, producción de textos de escritura personal en experiencias de trabajo individual y grupal (autobiografías), experiencias de oralidad individual (exposiciones, presentaciones, micro-conferencias) y grupal (charlas, mesas redondas, debates), actividades de role playing, mesas de encuentro entre docentes, familias y estudiantes; visitas de estudio y encuentros socio-profesionales.

\section{Prácticas educativas en el mundo socio productivo}

Estas experiencias se organizan a partir de un Plan de Búsqueda, una guía de trabajo que orienta a los estudiantes en la búsqueda de conocimientos en el ámbito económico, cultural, político, educativo, científico, tecnológico y/o artístico, en el que podrían insertarse en su práctica educativa. La idea es que, en la experiencia de la práctica, los jóvenes "hagan preguntas a la realidad laboral" e investiguen sobre ella en terreno ${ }^{9}$. Entre la variedad de prácticas educativas que se realizan, pueden mencionarse: prácticas educativas en el hogar y/o en un emprendimiento productivo familiar10, prácticas de acompañamiento a un oficio, ocupación, empleo o profesión, fuera de la escuela y del ámbito hogareño; pasantías educativas ${ }^{11}$, formación laboral y

9 Más allá de ofrecer a los estudiantes un espacio de conocimiento en la acción, estas prácticas consisten simbólicamente en un "paso", un ejercicio en un lugar de trabajo específico, con todas sus características y demandas: el cumplimiento de un horario determinado, el trabajo en equipo, la aceptación de una coordinación/dirección, la capacitación en servicio, etc. Por otra parte, tal como lo confirman numerosas experiencias y estudios, estas prácticas son fuente importante de motivación para los jóvenes.

10 Esta práctica recupera los antecedentes de las EFA (Escuelas de la Familia Agrícola). El estudiante permanece un período en la escuela y otro con la familia. Se trata de un modelo que permite y ofrece a los estudiantes la oportunidad de seguir estudiando en su propio medio, con el acompañamiento de la familia y sin emigrar a las grandes ciudades.

11 Las realiza el estudiante en organizaciones de su comunidad (ONG, empresas, instituciones públicas, organizaciones no gubernamentales entre otras), durante un tiempo determinado, bajo la organización y supervisión de la escuela, con el objeto de confrontar con la práctica los saberes teóricos adquiridos. Como señala Jacinto (2010), constituyen "un Acto Educativo (...) una actividad siempre curricular y supervisada por la institución escolar (...) deben guardar una estrecha relación con las finalidades formativas del plan de estudios, así como proporcionar oportunidades efectivas de participar en situaciones reales de vida y de trabajo en el contexto socio-productivo" (p. 124). De esta manera, la pasantía permite aplicar y desarrollar conocimientos y habilidades en contextos reales, articulando el ámbito educativo con el del trabajo, extendiendo la actividad de la escuela a las organizaciones de destino, y convirtiendo a estas últimas en ámbitos de aprendizaje concretos, en los que los pasantes se insertan 
emprendimientos escolares vinculados a la producción de bienes y/o servicios ${ }^{12}$.

\section{En cuanto a la evaluación}

Formación para la Vida y el Trabajo es un espacio curricular que demanda evaluar "el conocimiento en la acción". Por eso, si bien la evaluación del proceso es importante, no lo es menos la evaluación del resultado, habida cuenta del carácter práctico y funcionalista de toda acción. De este modo, dos son las miradas: una de ellas, sobre el proceso, o sea, evaluar el desarrollo del itinerario que va realizando el estudiante en las actividades propuestas, y dentro de él, la apropiación de los contenidos; la otra mirada se dirige a los resultados, lo que también conlleva la transferencia de lo aprendido a otras situaciones y contextos.

Articulando los procesos de enseñanza, aprendizaje y de evaluación, en Formación para la Vida y el Trabajo se concede una especial importancia a las tareas metacognitivas, que permiten a los jóvenes desocultar la manera en que están aprendiendo, para poder seguir haciéndolo más y mejor. Además, las secuencias de actividades incorporan siempre la evaluación crítica de los procesos y de las producciones, y las posibilidades para mejorarlas, es decir, el rediseño con vistas al perfeccionamiento, corrigiendo los defectos o deficiencias detectadas. Por otra parte, son objeto de evaluación permanente, tanto los aprendizajes disciplinares como aquellos vinculados a la formación para la ciudadanía activa y la reflexión en torno a la práctica solidaria.

En virtud de estas premisas, las experiencias que se están llevando a cabo en las instituciones educativas de la Provincia de Córdoba, Argentina, permiten visualizar la progresiva innovación y diversificación de los dispositivos e instrumentos de evaluación, con el propósito de que puedan dar cuenta de la complejidad de las situaciones pedagógicas que se presentan en el espacio curricular. Para ello, se están incorporando portafolios con trabajos e informes escritos, individuales o grupales; puestas en común con presentaciones individuales o colectivas; presentación de trabajos escritos o productos audiovisuales como instancias de cierre de las distintas etapas de aprendizaje; entrevistas a los estudiantes; jornadas de evaluación conjunta, entre estudiantes, docentes y demás actores participantes del proyecto formativo.

\section{Reflexiones finales}

Como afirma Krauskopf (2008), los jóvenes están en condiciones de "ser promotores del conocimiento de sus sociedades y sus comunidades para tener la oportunidad de investigar e incidir en las propuestas de sociedad con sus propias visiones y destrezas" (p. 175). Es necesario, entonces, generar las condiciones para que los estudiantes se sientan habilitados a participar, reconociéndose como sujetos constructores de las situaciones que viven y transformadores de las condiciones que los afectan.

Se constata una amplia aceptación del espacio curricular y de sus propuestas formativas, tanto por parte de los estudiantes y sus familias como de los docentes, así como del sector académico y productivo. Esta aceptación nos demanda, entonces, agudizar la mirada y los instrumentos de que disponemos para evaluar el proceso de implementación, así como potenciar las acciones de desarrollo curricular y acompañamiento a las instituciones y docentes. El gran desafío sigue siendo conocer los intereses, preocupaciones e inquietudes de los jóvenes, así como sus recorridos vitales, marcados muchas veces por los procesos de exclusión, y revisar las representaciones que de ellos tenemos.

con el objetivo de ampliar y afianzar su formación. Se encuadran jurídicamente en la Ley de Pasantías de la Provincia de Córdoba $N^{\circ}$ 8.477/1995, que actualmente se encuentra en revisión en virtud del Decreto Poder Ejecutivo Nacional No 13.74/2011. Régimen General de Pasantías del Nivel Secundario.

12 Estos emprendimientos revisten características diversas: son reales o simulados, con fines sociales y/o comunitarios, tecnológicos (referidos a la fabricación de un producto innovador), comerciales o productivos, culturales, solidarios, científicos, etc., y suelen organizarse bajo la forma de cooperativas, mutuales, clubes, o micro-emprendimientos escolares. 


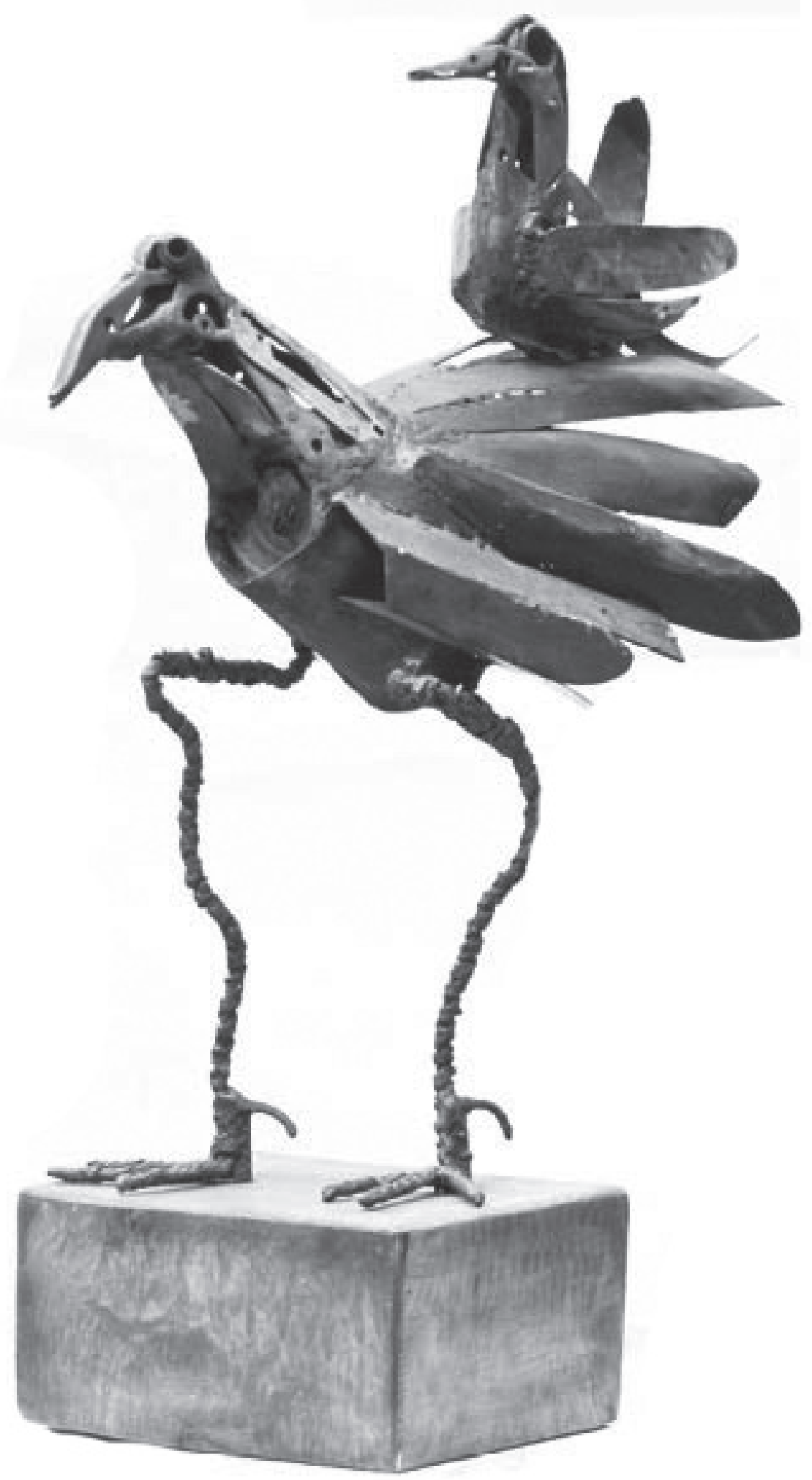

Artista: Felipe Ruiz

Dimensiones: $60 \times 50 \times 30 \mathrm{~cm}$

Tecnica: Escultura en metal

Año: 2010 
Aisenson, D. (1997). Perspectivas actuales en Orientación Vocacional. Ensayos y Experiencias, 3 (18), 7-19.

Aisenson, D. y Equipo de investigaciones en Psicología de la Orientación. (2002). Después de la Escuela. Buenos Aires: EUDEBA.

Argentina, Ministerio de Educación. (2011). Orientaciones para el desarrollo institucional de las propuestas sociocomunitarias solidarias. Buenos Aires: Autor.

Argentina, Ministerio de Educación. Consejo Federal de Educación. (2009). Lineamientos Políticos y Estratégicos de la Educación Secundaria Obligatoria. Versión Final. Resolución CFE Nº 84/09. Buenos Aires: Autor.

Borzese, C. (2008). Desde la práctica. Una metodología de formación para el trabajo con jóvenes de sectores vulnerables. Buenos Aires: Dunken.

Ferreyra H. (1996). Educación para el trabajo... trabajo en la educación. Buenos Aires: Novedades Educativas.

Ferreyra, H. A., Gallo, G. y Zecchini, A. (2006). Educar en la acción para aprender a emprender. Organización y gestión de proyectos socio-productivos y cooperativos. Buenos Aires: Novedades Educativas.

Gobierno de Córdoba. Ministerio de Educación. Secretaría de Educación. Subsecretaría de Promoción de Igualdad y Calidad Educativa (2011a). Educación Secundaria. Encuadre General 2011-2015. Córdoba, Argentina: Autor.

Gobierno de Córdoba. Ministerio de Educación. Secretaría de Educación. Subsecretaría de Promoción de Igualdad y Calidad Educativa (2011b). Diseño Curricular del Ciclo Básico de la Educación Secundaria 2011-2015. Córdoba, Argentina: Autor.

Heller, A. (1991). Sociología de la Vida Cotidiana. Barcelona, España: Península.
Jacinto, C. (2009). Consideraciones sobre estrategias de inclusión con calidad en la escuela secundaria. SITEAL, IIPE-UNESCO. Sede Regional Buenos Aires y OEl. Obtenido el 24 de Agosto de 2012, desde http://www.siteal.iipe-oei.org/contenido/58

Jacinto, C. (2010). Reformulaciones recientes acerca de la formación para el trabajo en la educación secundaria general. En IIPE. UNESCO. Educación y trabajo: articulaciones y políticas (pp.11-135). Buenos Aires: Autor. http://www.fediap.com.ar/ administracion/pdfs/Educacion \%20y\%20Trabajo\%20-\%20 Articulaciones $\% 20 y \% 20$ Pol\%C3\%ADticas $\% 20-\% 20$ IIPE $\% 20$ UNESCO.pdf (29 mayo, 2012).

Krauskopf, D. (2008). Dimensiones de la participación en las juventudes contemporáneas latinoamericanas. Santiago: FLACSO Chile.

Messina, G., Pieck, E. y Castañeda, E. (2008). Educación y Trabajo. Lecciones desde la práctica innovadora en América Latina. Santiago: UNESCO. Obtenido el 29 de Septiembre de 2011, desde http://unesdoc.unesco.org/images/0016/001608/160881s.pdf

Perkins, D. (2010). El aprendizaje pleno. Principios de la enseñanza para transformar la educación. Buenos Aires: Paidós.

UNESCO (2007). Educación de calidad para todos. Un asunto de derechos humanos. Documento de discusión sobre políticas educativas en el marco de la II Reunión Intergubernamental del Proyecto Regional de Educación para América Latina y el Caribe. Buenos Aires: Oficina Regional de Educación para América Latina y el Caribe (OREALC/UNESCO). Obtenido el 29 de Mayo de 2012, desde http://unesdoc.unesco.org/ images/0015/001502/150272s.pdf

Valdés, T. (1998). El Secreto de la Pirámide. Algunas ideas para la cualificación de la función docente en los sistemas de educación para el trabajo. Biblioteca Digital OEI. Cuaderno de Trabajo 3. Obtenido el 5 de Junio de 2012, desde http://www.oei.es/oeivirt/ $\mathrm{fp} / \mathrm{cuad} 3 \mathrm{a} 04 . \mathrm{htm}$ 